\title{
A detailed kinetic study of the direct photooxidation of 2,4,6-trichlorophenol
}

\author{
Jose Ángel Pino-Chamorro, Tamás Ditrói, Gábor Lente*, István Fábián \\ Department of Inorganic and Analytical Chemistry, University of Debrecen, P.O.B. 21, H-4010, Debrecen, Hungary
}

\section{A R T I C L E I N F O}

\section{Article history:}

Received 26 January 2016

Received in revised form 26 July 2016

Accepted 26 July 2016

Available online 27 July 2016

\section{Keywords:}

Quantitative photochemistry

Light intensity dependence

Chlorophenols

Temperature dependence

Photochemical mechanism

Kinetics

Photodegradation

2,4,6-trichlorophenol

Polychromatic light

Mechanism of reaction

Quantum yield

\begin{abstract}
A B S T R A C T
The direct photodegradation of 2,4,6-trichlorophenol (TCP) by UV-vis light was studied in aqueous solution in order to analyze the mechanism of the photochemical process and to determine the kinetic parameters including the quantum yield. Based on initial rate studies at different overall volumes and illumination patterns, it was proved that the rate of the process is directly proportional to the intensity of irradiating light. A significant, but moderate acceleration of the reaction rate with increasing temperature was revealed between 5.0 and $35.0^{\circ} \mathrm{C}$, which could be interpreted readily by assuming that the excited state of TCP is involved in two competing processes. High pressure liquid chromatography and mass spectrometry provided us information on the nature of the intermediates and the products formed. 2,6Dichloro-1,4-benzoquinone, 3,5-dichloro-2-hydroxy-1,4-benzoquinone and 2,6-dihclorohydroxyquinone were detected as products and/or intermediates, and there were also hints of the formation of 3,5-dichlorobenzene-1,2-diol and 3,5-dichloro-1,2-benzoquinone. A possible degradation mechanism is proposed to interpret the kinetic findings.
\end{abstract}

(c) 2016 Elsevier B.V. All rights reserved.

\section{Introduction}

Chlorophenols are common and persistent environmental pollutants produced in a number of industrial processes. They are not decomposed by usual waste water treatment techniques, and the development of suitable degradation methods has attracted the attention of a large and diverse research community [1-22]. The use a specifically designed iron complexes for the activation of hydrogen peroxide seems to be a particularly successful strategy $[2,7,11]$. In these cases, no dioxins are produced among the oxidation products [7], which is a major concern with other waste treatment methods, for example the direct incineration of solid chlorophenols. The use of ozone [5,6], Fenton's reagent [5], peroxidase enzyme [13], electrooxidation [21], or even simple absorption processes [22] are also possible alternatives in the abatement of chlorophenol-containing waste.

Illumination with UV or visible light can also assist such processes. This is typically done using heterogeneous photocatalysts such as $\mathrm{TiO}_{2}[1,15,19]$, spinels $[18,20]$ and $\mathrm{g}-\mathrm{C}_{3} \mathrm{~N}_{4}$ [17]. Because of the limited light absorption of chlorophenols (which all

\footnotetext{
* Corresponding author.

E-mail address: lenteg@science.unideb.hu (G. Lente).
}

form colorless solutions), their direct photolysis was a lot less intensely investigated [3-5]. The iodine-sensitized photooxidation was also considered as a possible alternative [14]. An interesting and unintuitive observation was also made during a kinetic study of the chemical oxidation of chlorophenols: the rate of reaction is influenced by light, even if it is in the visible range [8]. The reason for this phenomenon was understood to be the photochemical transformations of quinones, which form as intermediates in chlorophenol oxidation $[8,23,24]$. Therefore, an adequate kinetic and mechanistic study of the chemical oxidation of chlorophenols must be done in the dark in order to separate the thermal and photolytic components of the process [9,11,12].

The accelerating effect of light on chlorophenol oxidation also implies that their photochemical properties must be thoroughly investigated together with the known intermediates of the process. Recently, experimental techniques were developed for the online monitoring of photochemical processes using diode array spectrophotometers alone or with external light sources [9,25-29]. These techniques use online monitoring and can yield kinetic data on photoreactions that have very high time resolution and thus be evaluated by up-to-date kinetic analysis methods. They facilitated detailed studies on direct photolytic processes $[23,29]$ and photochemically initiated chain reactions [25]. The 
experience gained in a number of different studies was summarized in a review article a few years ago [26].

In this paper, we report a detailed kinetic study on the photochemical reaction of 2,4,6-trichlorophenol, which has the widest industrial applications and is the most important among the chlorophenols $[7,11,14]$. A new, custom-built photoreactor was used to carry out this quantitative study, which improves the applicability of online monitoring of photochemical reactions compared to the earlier instruments used for this purpose [26].

\section{Experimental section}

\subsection{Materials}

Solid 2,4,6-trichlorophenol (TCP) was purchased from SigmaAldrich and used as received. The concentration of TCP in our experiments was typically around $2.0 \times 10^{-4} \mathrm{M}$. All the experiments were carried out in $0.010 \mathrm{M}$ phosphate and borax buffers at $25.0^{\circ} \mathrm{C}$ (except some experiments to study the dependence on temperature). The buffer stock solutions were prepared using sodium phosphate dihydrate (Reanal) and titrated to $\mathrm{pH} 7.04$ with $\mathrm{NaOH}$ purchased from VWR, and using disodium tetraborate (Reanal) at pH 9.2. All stock solutions and samples were prepared with double deionized, ultrafiltered and distilled water from a Millipore Q system.

\subsection{Instruments and software}

The excitation light source (AvaLight-LDXE), the detecting light source (AvaLight DHc), the detector (Avaspec-ULS2048L-TEC-RS) and all other optical cables of the photoreactor used in this study were purchased from Avantes, Netherlands. The light source (excitation lamp) has a maximum brightness of $385 \mathrm{~mW}$ optical output in the $170-1100 \mathrm{~nm}$ range (Fig. S1 in the Supplementary information). A FOS-1-Inline fiber optic switch was used, which allowed us to switch on and off the focused polychromatic light beam that provided the driving source of the photoreaction. Moreover, the halogen and deuterium lamps of the detecting light source can be independently switched on and off. The sample holder used was equipped with a temperature controller Peltier unit configured for absorbance measurement. The cell holder was modified with vertically movable detection ports to avoid scattered or fluorescent light entering the detector. The spectrophotometer (detector) used was AvaSpec-ULS2048L-TEC-RS, which is an internally cooled CCD detector with variable slit size. Standard four-sided quartz cuvettes (optical path length: $1.000 \mathrm{~cm}$ ) were used in all experiments. The solution in the cuvette was stirred using a $4 \mathrm{~mm} \times 2 \mathrm{~mm} \times 2 \mathrm{~mm}$ Teflon-coated stirring rod with the built-in magnetic stirrer of the sample holder to keep the solutions homogeneous at all times. The detailed description and performance characterization of this novel photoreactor was published in a recent technical note [30].

Ferrioxalate actinometry was performed following a published method [28]. A flow of nitrogen gas was circulated through the cell holder to avoid the condensation of water in the walls of the cell during the temperature dependence study. In these experiments, the cuvette was kept in the sample holder during $10 \mathrm{~min}$ before starting the experiments to ensure that the solution temperature was homogeneous. Kinetic traces were fitted and other least squares analyses were carried out by the software Scientist [31]. Particularly, initial rates of absorbance change ( $v_{0}$ values) were determined by using a polynomial probe function to fit the experimental kinetic trace. The coefficient of the first order term of the polynomial gives the initial rate in this process. Further details of this method can be found in the literature of chemical kinetics [32].
TCP and its degradation products were separated and analyzed by a Shimadzu LC-10AP HPLC system equipped with a Shimadzu SPD-M10A diode array detector, a Luna C18 (2) $(3 \mu \mathrm{m})$ $100 \times 4.6 \mathrm{~mm}$ reverse phase column and using 7:3 (V/V) mixture of methanol and water as the mobile phase. The flow rate was set at $1.0 \mathrm{~mL} / \mathrm{min}$. The intermediates formed from TCP degradation were identified by comparing their retention time and spectrum with the external standards purchased from commercial sources. Under the conditions described, the retention times of the external standards were: TCP: $10.65 \mathrm{~min}, 2$,6-dichloroquinone (DCQ): $2.75 \mathrm{~min}$.

ESI-MS measurements were carried out with a Bruker micrO$\mathrm{TOF}_{\mathrm{Q}}$ mass spectrometer equipped with a quadrupole and time-offlight (Q-TOF) analyzer in positive ion mode. The nebulizer pressure was set to 6 psi ( 0.41 bar), $\mathrm{N}_{2}$ was used as drying gas at $4 \mathrm{~L} / \mathrm{min}$, the temperature was maintained at $160^{\circ} \mathrm{C}$, and the applied capillary voltage was $-3.0 \mathrm{kV}$. The typical error of mass measurement was below 0.005 in the $m / z$ range from 80 to 600 . Spectra were accumulated and recorded by a digitizer at a rate of $2 \mathrm{GHz}$.

\section{Results and discussion}

\subsection{Preliminary observations}

In the $\mathrm{pH}$ range used in the present study ( $\mathrm{pH}=7.04-9.2), 2,4,6-$ trichlorophenol has two UV bands centered at 254 and $312 \mathrm{~nm}$ that are characteristic of the dissociated anionic form $\left(\mathrm{p} K_{\mathrm{a}}=6.15\right)[9,12]$. The initial observations confirm that the photoreaction can be driven even by a commercial diode array spectrophotometer, the photochemical use of which is now well documented [26]. The photodegradation of TCP was followed in a custom built photoreactor as descibred in the Experimental section. A cuvette containing a solution of TCP was illuminated and the UV-vis absorption spectrum was scanned repeatedly in the range of 230$700 \mathrm{~nm}$ at $\mathrm{pH} 7.05$ and 9.25 using phosphate and borax buffers at $30 \mathrm{~s}$ intervals. The photooxidation of TCP at these two $\mathrm{pH}$ values had similar rates. An earlier research report found a large difference between the TCP oxidation rates at $\mathrm{pH} 7.4$ and 9.0 [13]. However, that must have been caused by the properties of the peroxidase oxidant used in that study [13] as TCP itself undergoes no major change in this $\mathrm{pH}$ region (its $\mathrm{p} K_{\mathrm{a}}$ is 6.15). Figs. S2 and S3 show experiments in the two different media and also confirm an important point: the kinetic observations do not depend on the concentration of the buffer used in any significant way, so the buffers are most probably innocent in this case.

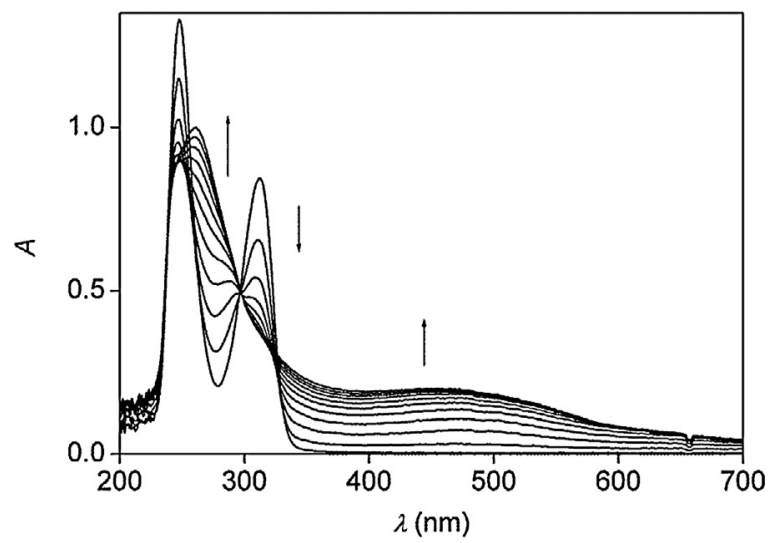

Fig. 1. Typical spectral changes in an irradiated aqueous solution of 2,4,6-TCP. $[\mathrm{TCP}]=0.2 \mathrm{mM}$; [phosphate buffer] $=0.010 \mathrm{M}$; path length $1.000 \mathrm{~cm} ; V=3.00 \mathrm{~cm}^{3}$; $T=25.0^{\circ} \mathrm{C}$; overall reaction time: $\left.300 \mathrm{~min}\right)$. 
Typical time-resolved spectra measured during the photoreaction of TCP are shown in Fig. 1. Only spectra obtained by $30 \mathrm{~min}$ intervals are plotted in the figure for clarity, but the time resolution of the original data set is much higher. Literature results indicate that these bands correspond to the formation of DCQ [14,16], which is one of the products of the process. Its subsequent transformation into a mixture of 2,6-dichloro-hydroquinone (DCHQ) and DCHB $[23,26]$ is a known photoreaction, but DCHB may in fact also form as the immediate product of the photochemical oxidation of TCP. The reactants and products could be monitored with considerable selectivity because the absorption bands of 2,6-dichloroquinone (DCQ, yellow) and 3,5-dichloro-2-hydroxybenzoquinone (DCHB, purple) were reasonably separated from TCP (colorless, only UV absorption). The oxidation involves the appearance of two bands centered at ca. 260 and $480 \mathrm{~nm}$ after $300 \mathrm{~min}$. A very slow disappearance of the band at $480 \mathrm{~nm}$ was observed after $24 \mathrm{~h}$. Fig. 2 shows the kinetic traces at 260, 312 and $480 \mathrm{~nm}$ in a five-hour experiment. The trace at $260 \mathrm{~nm}$ clearly displays a small initial decrease in the absorbance and the curve recorded at $480 \mathrm{~nm}$ also hints at an induction period. Therefore, it can be concluded that there is at least one primary and at least one secondary photoproduct.

In addition to the literature data already mentioned $[14,16,23,26]$, other researchers reported the presence of some different intermediates in the process of TCP photodegradation [17]. Thus, in order to verify the nature of the compounds in the reaction mixture, HPLC and mass spectrometry was applied to obtain qualitative information on this system.

\subsection{HPLC experiments}

The degradation products of TCP were monitored using HPLC with UV-vis detection. The HPLC chromatograms and UV-vis spectra of the solutions are shown in Fig. 3 (after $1 \mathrm{~h}$ of illumination) and Fig. 4 (after $5 \mathrm{~h}$ of illumination). The final products from TCP degradation were identified primarily by comparing the UV-vis spectra with those of pure compounds (Figs. S4 and S5 in the Supplementary information). The Uv-vis spectra of TCP $[9,12,13]$, DCQ $[9,12,13,23,24]$ and DCHB $[23,24]$ are all available from the literature.

After $1 \mathrm{~h}$ of irradiation, the chromatogram shows the appearance of two peaks with retention times 2.29 and 3.93 min together with the TCP peak with retention time $9.86 \mathrm{~min}$. After $5 \mathrm{~h}$, practically complete degradation of TCP was observed. An increase in the peak at $2.29 \mathrm{~min}$ and a decrease in the peak at $3.92 \mathrm{~min}$ were

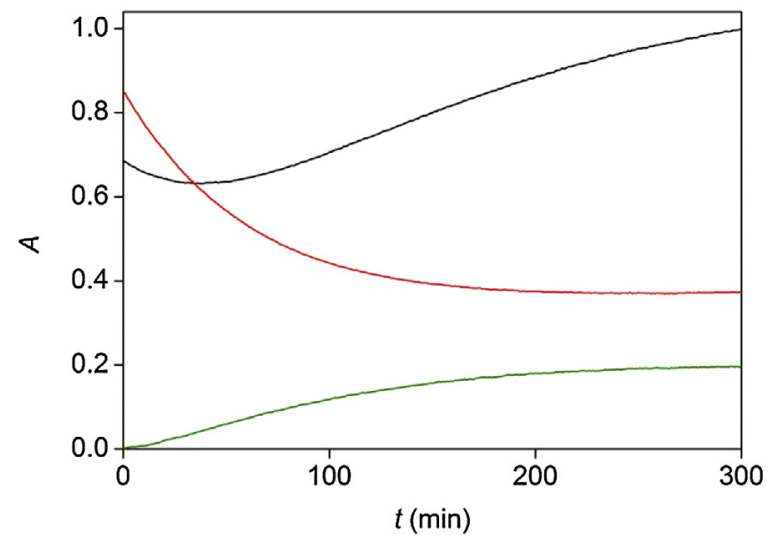

Fig. 2. Absorbance changes as a function of time in an irradiated aqueous solution of TCP at selected wavelengths. The wavelengths chosen are the maximum of DCQ (black line, $260 \mathrm{~nm}$ ), TCP (red line, $312 \mathrm{~nm}$ ) and DCHB (green line, $480 \mathrm{~nm}$ ). Conditions are the same as in Fig. 1. (For interpretation of the references to colour in this figure legend, the reader is referred to the web version of this article.). also detected. These two signals could probably be assigned to 2,6dichloro-hydroquinone (DCHQ) and 3,5-dichlorobenzene-1,2-diol. Moreover, an interesting observation was the appearance of a new peak at $1.62 \mathrm{~min}$. This compound has a band centered at $260 \mathrm{~nm}$, which is identical with the observed band for the final product detected in the photoreactor and could indicate the presence of 3,5-dichloro-1,2-benzoquinone. More explanation about these compounds will be given in later sections.

\subsection{ESI-MS experiments}

ESI-MS spectra were also used to identify the photodegradation products of TCP. The ESI mass spectrum of TCP after $1 \mathrm{~h}$ irradiation shows the presence of two signals, which can be identified based on the peak $\mathrm{m} / \mathrm{z}$ values and the characteristic isotopic patterns (Fig. S6). The first is centered at $m / z 176.9$ and could be assigned to 2,6-dichloro-hydroquinone (DCHQ) and probably also to 3,5-dichlorobenzene-1,2-diol, which has the same $\mathrm{m} / \mathrm{z}$ value. These two compounds might correspond to peaks 2 and 3 in the chromatogram shown in Fig. 3. The peak at $m / z=194.9$ is attributed to and TCP. Other compounds were not observed in the mass spectrum after $5 \mathrm{~h}$ (Fig. S7). It is possible that the ESI technique is not able to ionize quinones such as DCQ 3,5-dichloro-2-hydroxy1,4-benzoquinone (DCHB) or 3,5-dichloro-1,2-benzoquinone, which have been identified previously in this reaction as products [23]. Similar ionization problems have already been reported with studies involving 2,4-dichlorophenoxybutanoic acid and 2,4,5trichlorophenoxyacetic acid [33,34].

\subsection{Kinetic studies}

First, it is important to note that due to partial acid dissociation of chlorophenols in aqueous solutions, the reaction schemes of the TCP degradation need to account for the acid-base properties of this molecule. The $\mathrm{p} K_{\mathrm{a}}$ of TCP is 6.15 , and the Uv-vis spectra of the protonated and dissociated forms are different. [12] So, in principle the reaction pathways including the protonated (neutral) and dissociated (anionic) forms may contribute to the overall reaction rate. Since our aim was to use conditions that were close to the possible applications in water treatment technologies (neutral $\mathrm{pH}$ ), we set $\mathrm{pH}=7.04$ with a phosphate buffer in the majority of the experiments reported here. Under these conditions, about $88 \%$ of TCP is in the dissociated (phenolate) form [6]. This form also happens to display much more substantial absorption in the wavelength range of illumination than the neutral TCP molecule. Therefore, for all practical purposes, it was sufficient to assume that our observations provide information on the photoreactions of the phenolate ion. In the rest of the paper, the common convention of coordination chemistry will be used: the abbreviation TCP will refer collectively to the two different protonated forms of 2,4,6trichlorophenol with the understanding that the $\mathrm{pH}$-dependent speciation must be kept in mind at each specified $\mathrm{pH}$.

In Fig. 2, the kinetic trace at $312 \mathrm{~nm}$ reflects primarily the concentration change of TCP. However, the contributions of other species to the absorbance at this wavelength are non-negligible as evidenced by the non-zero final absorbance seen in Fig. 2. This trace could be fitted to a pseudo-first order expression (exponential curve) with an acceptable precision and the observed rate constant was determined to be $k=(1.82 \pm 0.01) \times 10^{-2} \mathrm{~min}^{-1}$. The good fit and the value are both in reasonable agreement with the observations reported by Shen et al. at the same $\mathrm{pH}$ [3], especially if the different illumination intensities are taken into account. However, it should be noted that a true pseudo-first order process should give identical rate constants at all wavelengths of monitoring [32]. Furthermore, a homogenous photochemical process is actually never expected to have a simple first order 

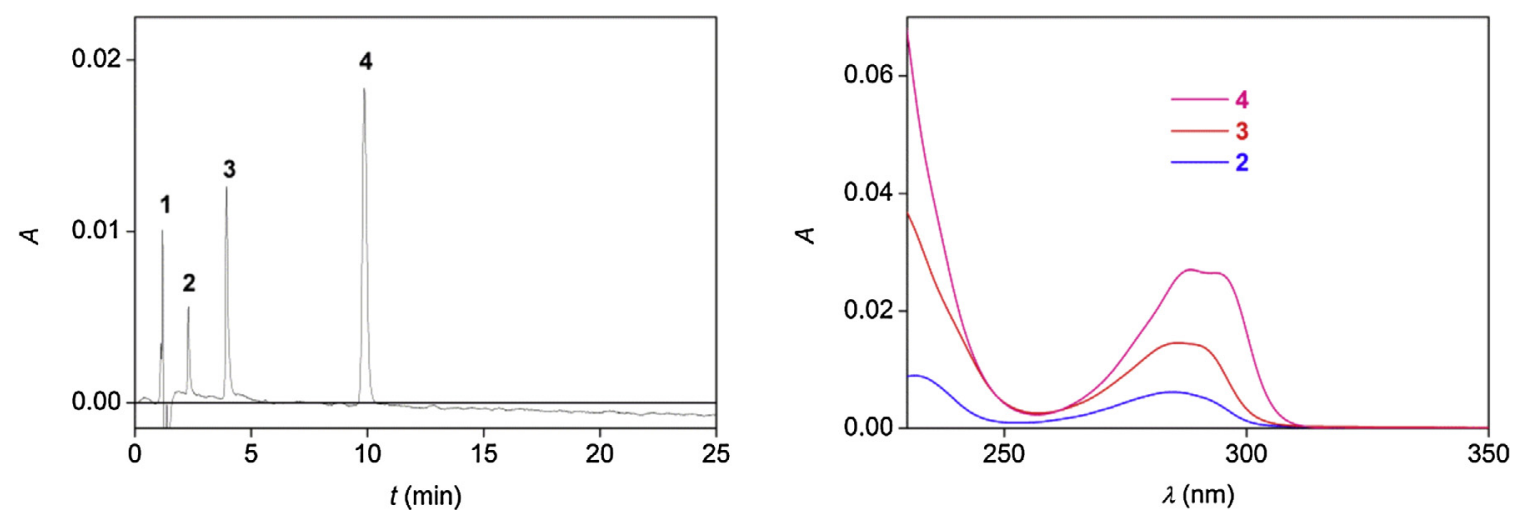

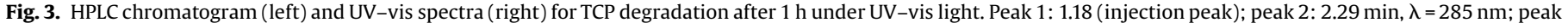
3: $3.93 \mathrm{~min}, \lambda=287 \mathrm{~nm}$; peak 4: $9.87 \mathrm{~min}, \lambda=287$ and $293 \mathrm{~nm}$. [TCP $]_{0}=0.20 \mathrm{mM}$, [phosphate buffer] $=0.010 \mathrm{M}$.
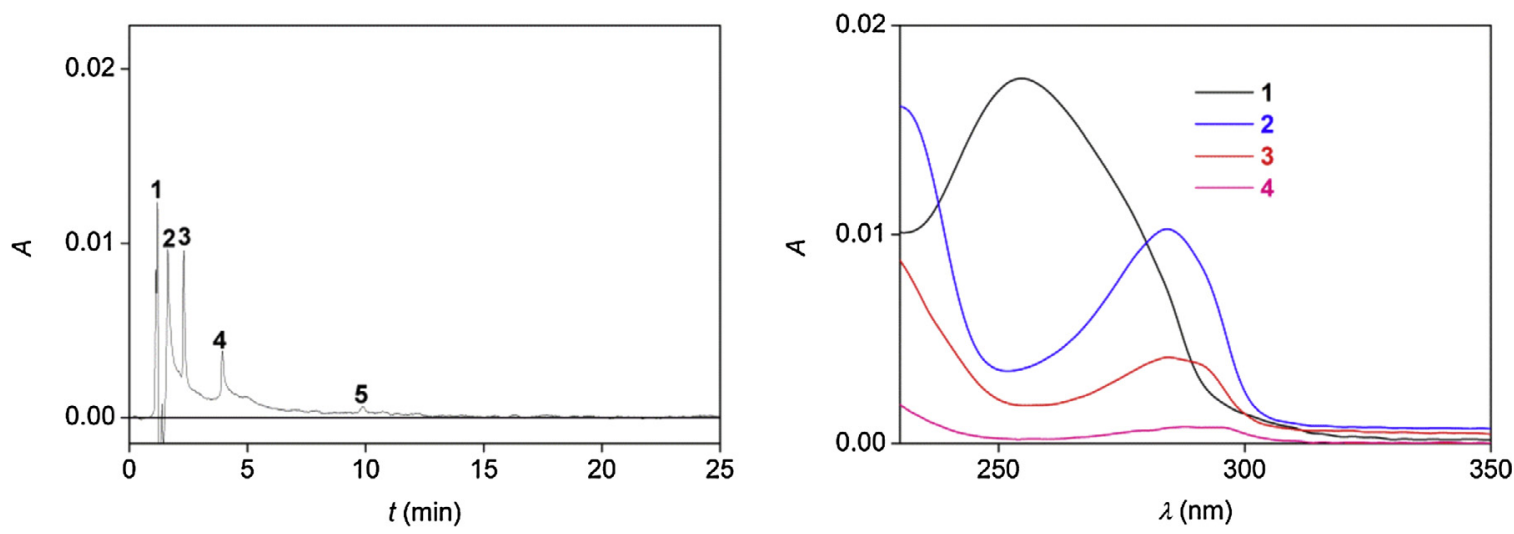

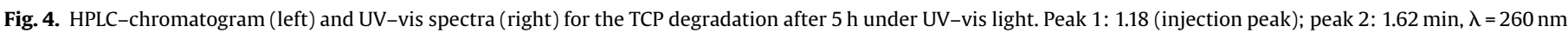
peak 3: $2.29 \mathrm{~min}, \lambda=285 \mathrm{~nm}$; peak 4: $3.92 \mathrm{~min}, \lambda=287 \mathrm{~nm}$; peak 5: $9.88 \mathrm{~min}, \lambda=287$ and $293 \mathrm{~nm}$. $[\mathrm{TCP}]_{0}=0.20 \mathrm{mM}$, [phosphate buffer] $=0.010 \mathrm{M}$.

rate equation. Even the simplest photochemical transformation has the following rate expression [23,27,32]:

$\frac{d[\mathrm{~A}]}{d t}=-\int \frac{\Phi_{P, \lambda} \Phi_{\lambda}}{V}\left(1-10^{-\varepsilon_{\mathrm{A}, \lambda} l[\mathrm{~A}]-\sum \varepsilon_{i, \lambda} l\left[\mathrm{~B}_{i}\right]}\right) \frac{\varepsilon_{\mathrm{A}, \lambda}[\mathrm{A}]}{\varepsilon_{\mathrm{A}, \lambda}[\mathrm{A}]+\sum \varepsilon_{i, \lambda}\left[\mathrm{B}_{i}\right]} d \lambda$

In this equation, $\mathrm{A}$ is the photoactive species, $\mathrm{B}_{i}$ are various nonphotoactive species that absorb light, $\Phi_{\mathrm{P}, \lambda}$ is the spectral photon flux of the illumination, $\Phi_{\lambda}$ is the differential quantum yield, $V$ is the volume of the reactor, $l$ is the optical path length, whereas $\varepsilon_{\mathrm{A}, \lambda}$ and $\varepsilon_{i, \lambda}$ are the molar absorption coefficients of the respective species. The terminology used here follows the current recommendations of IUPAC [35]. Given the complexity of Eq. (1), it must be concluded that the fact that the kinetic traces are very close to pseudo-first order at $310 \mathrm{~nm}$ is a consequence of coincidences involving rate constants and absorption coefficients, thus, the information content of the determined observed rate constant is very complex. To avoid these problems, only initial rates were used in the rest of this study as a source of quantitative information about the rate equation of the photodegradation.

In order to explore how the intensity of light affects the kinetics of the reaction, we have carried out different experiments, in which the reaction volume was changed at constant irradiating light flux. Through the volume change, the number of photons absorbed in unit volume can be influenced and its controlled variation can be used to study the dependence of the reaction rate on the illumination intensity. This fact has been explained previously in several recent publications from our group $[23,25,26,29]$. The volume of the sample was changed from 1.0 to $3.5 \mathrm{~cm}^{3}$ in a standard fluorometric cuvette (path length: $1.000 \times 1.000 \mathrm{~cm}$ ), i.e., the light intensity per volume can be varied by a factor of 3.5 . This is substantially larger than the ranges in previous studies $[23,25,29]$ and is an advantageous aspect of the photoreactor used in this study.

The initial rate of absorbance change $\left(v_{0}\right)$ for TCP degradation was measured using the data obtained during the first $30 \mathrm{~min}$ of reaction at $312 \mathrm{~nm}$ and is shown as a function of the volume of the sample in Fig. 5 [26]. The initial rate clearly decreases when the sample volume increases, and the measured points fit to a hyperbola very well, implying an inverse proportionality between

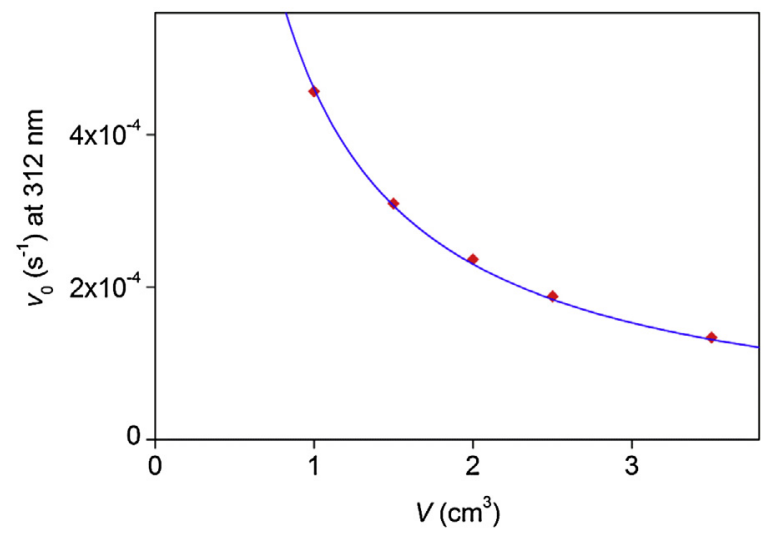

Fig. 5. Dependence of the initial rates the sample volume at $312 \mathrm{~nm}$ in the photodegradation of TCP. $[\mathrm{TCP}]=0.20 \mathrm{mM}$; [phosphate buffer $]=0.010 \mathrm{M} ; T=25.0^{\circ} \mathrm{C}$. 
the rate and the volume. A logarithmic version of this plot is shown in the Supplementary information as Fig. S8, where the points define a straight line with a slope of $-0.98 \pm 0.01$. As known from previous literature $[23,25,26]$, these observations prove that the reaction rate is directly proportional to the intensity of illumination.

To gain more information about this process, experiments with interrupted illumination were performed where the illumination ratio is defined as the time of illumination relative to the total time of reaction. [25,26] The average reaction rate is expected to be strictly proportional to the illumination ratio in a purely photochemical reaction. The measurements in this series differed in the duration of illumination $\left(t_{\mathrm{i}}\right)$ and dark periods $\left(t_{\mathrm{d}}\right)$ by opening and closing the shutter for controlled periods. In this way, the duration of a full cycle, $t_{\mathrm{c}}$, is given as $t_{\mathrm{c}}=t_{\mathrm{i}}+t_{\mathrm{d}}$. The cycles of illuminated and dark periods are repeated. Therefore, time values $t_{\mathrm{i}}$ and $t_{\mathrm{d}}$ define an illumination pattern.

Kinetic traces recorded during the experiments using different illumination patterns are shown in Fig. 6, where degradation of TCP was followed by using continuous illumination $\left(t_{\mathrm{i}}=50 \mathrm{~s}, t_{\mathrm{d}}=0 \mathrm{~s}\right.$; red trace) or a pattern with $10 \%$ illumination ratio $\left(t_{\mathrm{i}}=5 \mathrm{~s}, t_{\mathrm{d}}=45 \mathrm{~s}\right.$; black trace). For the quantitative evaluation of the experimental data, the average rate of absorbance change is used. In agreement with our expectation, the average initial rate value decreases with increasing the length the dark periods while keeping the cycle time constant. In fact, the average initial rates of the two kinetic experiments mentioned (red and black curves) differ by a factor of 10. Further experiments confirmed the direct proportionality between the illumination ratio and the reaction rate, as shown in Fig. 7. This finding indicates that the studied process is a relatively simple, direct photochemical transformation without a considerable parallel thermal pathways (dark reactions) or the involvement of photoinitiated chain processes. Should these latter factors contribute to the observations, more complex dependencies would be expected in the plot shown in Fig. 7 [25,26]. Fig. 6 also shows that the reaction is not driven by only visible light because the absorbance change is marginal when a cut-off filter is inserted into the excitation light beam, which eliminates light below $395 \mathrm{~nm}$ (green trace). Moreover, it is notable that the absence of stirring results in irreproducible, badly defined traces as inhomogeneity develops in the solution (blue trace in Fig. 6). This effect and the need for stirring in this type of photoreactor were emphasized in an earlier publication [23].

In order to analyze the photoreaction driven by polychromatic light in a quantitative way, it is very important to measure the

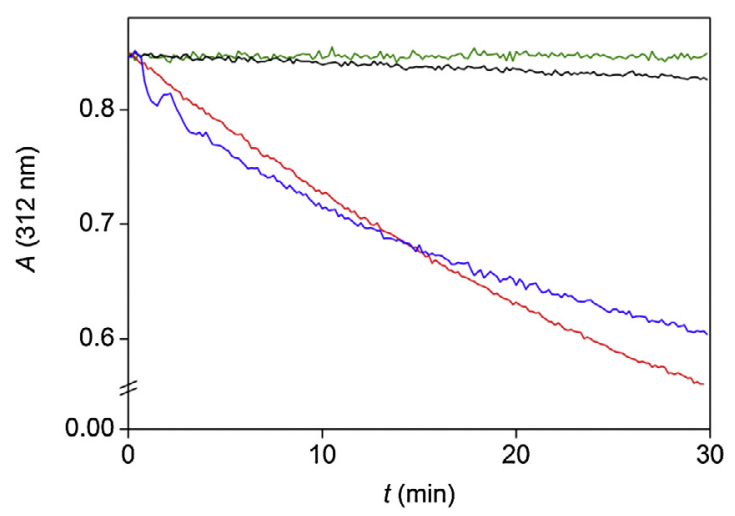

Fig. 6. Kinetic traces measured in the photochemical oxidation of TCP. $[\mathrm{TCP}]=$ $0.20 \mathrm{mM}$; [phosphate buffer] $=0.010 \mathrm{M}$; path length $1.000 \mathrm{~cm} ; V=2.00 \mathrm{~cm}^{3}$; $T=25.0^{\circ} \mathrm{C}$; only the visible light was used (filter cut-off at $395 \mathrm{~nm}$ ) (green trace); $10 \%$ illumination ratio (black trace); $100 \%$ illumination ratio (red trace); and no stirring (blue trace). (For interpretation of the references to colour in this figure legend, the reader is referred to the web version of this article.).

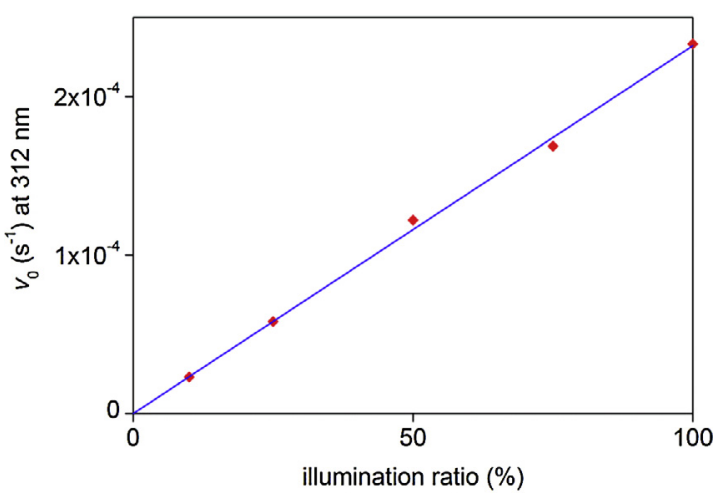

Fig. 7. Dependence of the average initial rates on the illumination ratio for the photodegradation of TCP. $[$ TCP] $=0.20 \mathrm{mM}$; [phosphate buffer] $=0.010 \mathrm{M}$; path length $1.000 \mathrm{~cm} ; V=2.00 \mathrm{~cm}^{3} ; T=25.0^{\circ} \mathrm{C}$.

relative intensity of the exciting light at each wavelength. For this purpose, we used an Analytik Jena SPECORD S600 diode array spectrophotometer. The relative energy spectrum of the excitation lamp was recorded and converted to an absolute scale by routine ferrioxalate actinometry [28]. The intensity spectrum of the excitation light is given in Fig. S1 of the Supplementary information. The total photon flux of the illumination was determined to be $\Phi_{\mathrm{P}}=62 \mathrm{nmol} / \mathrm{s}$.

The photon count $(N)$ in a photochemical experiment was defined in one of earlier works as the part of the photon flux absorbed by the photoactive species [23]. In the present case, the initial photon count is especially easily calculated from the intensity spectrum of the excitation light and the measured absorbance values of the studied solution because TCP, the photoactive species, is the sole absorbing species in the initial solution. A plot of reaction rates as a function of photon count is suitable for determining the quantum yield in a series of experiments where the initial concentration of the photoactive species is varied [23]. Fig. 8 shows that this plot is linear for the process studied here, as expected based on earlier observations about the direct proportionality between the rate and light intensity.

A straightforward method for the calculation of quantum yields based on this plot was already published [23]. The slope $(\alpha)$ of the fitted line in Fig. $8,(1.13 \pm 0.03) \times 10^{-4} \mathrm{nmol}^{-1}$, the volume of the reactor $(V)$ and the molar absorption coefficients $(\varepsilon)$ of the reactants and products need to be used in the following manner:

$$
\Phi=\frac{\alpha V}{\left(\varepsilon_{\mathrm{TCP}}-\varepsilon_{\mathrm{DCQ}}\right) l}
$$

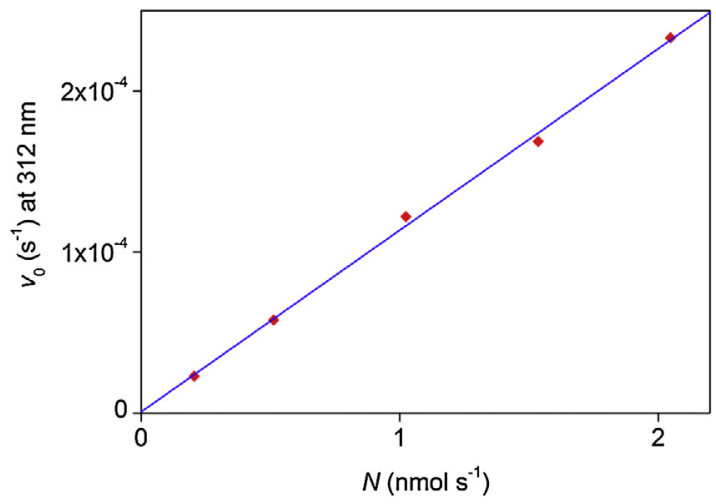

Fig. 8. Initial rate at $312 \mathrm{~nm}$ in the aqueous photoreaction of TCP as a function of photon counts. $[\mathrm{TCP}]=0.20 \mathrm{mM}$; [phosphate buffer $]=0.010 \mathrm{M}$; path length $=1.000$ $\mathrm{cm} ; V=2.00 \mathrm{~cm}^{3} ; T=25.0^{\circ} \mathrm{C}$. 
The molar absorbance for TCP $\left(\varepsilon_{\mathrm{TCP}}=4202 \pm 87 \mathrm{M}^{-1} \mathrm{~cm}^{-1}\right)$ and for DCQ $\left(\varepsilon_{\mathrm{DCQ}}=307 \pm 9 \mathrm{M}^{-1} \mathrm{~cm}^{-1}\right)$ were determined experimentally at $312 \mathrm{~nm}$ by measuring the UV-vis spectra of TCP and DCQ solutions in known concentration, respectively. These values agree well with previously reported ones $[10,13]$. The quantum yield $\Phi=0.057$ was calculated for TCP degradation as a result. This value is in agreement with those calculated in previous studies under different conditions $[4,5,14]$.

Another possible factor to be considered in a photochemical process is the dependence on the temperature. In this manner, a detailed study in the range of temperatures between 5.0 and $35.0^{\circ} \mathrm{C}$ was performed. Fig. 9 shows the absorbance-time traces at different temperatures. The decomposition occurs faster with increasing temperature, which may be somewhat unexpected as the rates of simple photochemical reactions at constant reactant compositions depend only on the illumination intensity but not on temperature. The photon counts are unchanged as the absorption spectra of TCP and DCQ are not influenced by this minor change in temperature. This fact can be explained by an increase of the quantum yield of a photochemical process when the temperature is raised [5]. Actually, the low value of the quantum yield (0.057) leaves the possibility of a temperature-dependent increase open. The phenomenon is readily interpreted by assuming that the excited form of TCP formed after light absorption is not only involved in a chemical reaction, but an energy loss not resulting in net chemical change (fluorescence, vibrational relaxation, an internal conversion followed by other processes, etc.) is also operative. This energy loss is most probably radiationless, as independent experiments showed that TCP practically does not show any fluorescence in aqueous solution (see Fig. S9 in the Supplementary information). Therefore, the reaction is very likely to obey the set of reactions:

$\mathrm{TCP} \stackrel{h v}{\rightarrow} \mathrm{TCP} * \mathrm{TCP} * \stackrel{k_{1}}{\rightarrow} \mathrm{TCPTCP} * \stackrel{k_{2}}{\rightarrow} \cdots \rightarrow \mathrm{DCQ}$

In this scheme, rate constant $k_{1}$ represents radiationless energy loss, whereas $k_{2}$ is the rate constant of the pathway that eventually leads to the formation of DCQ.

The dependence of the initial rates on the temperature is illustrated in Fig. 10. In this plot, an important effect of the temperature was observed. As the temperature increased from 5.0 to $35.0^{\circ} \mathrm{C}$, the rate of TCP degradation increased to 2.14 -fold. Following some literature precedents [36-41], this dependence was interpreted by assuming that both $k_{1}$ and $k_{2}$ have Arrhenius type temperature dependencies with pre-exponential factors $A_{1}$

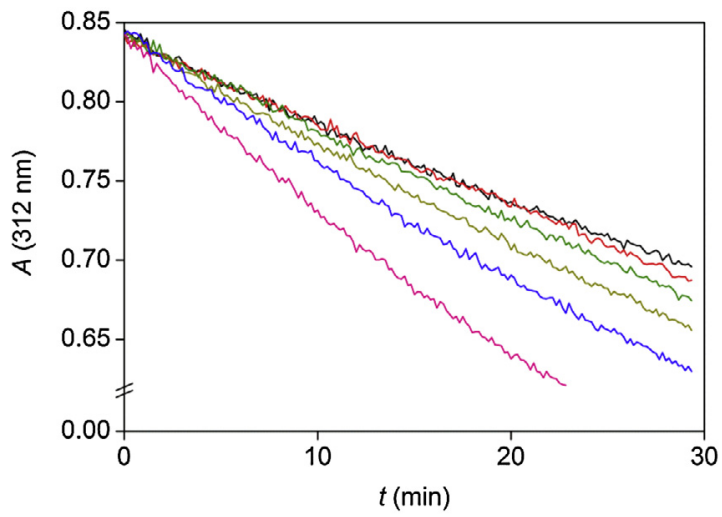

Fig. 9. Kinetic traces measured in the photochemical oxidation of TCP. $[\mathrm{TCP}]=$ $0.20 \mathrm{mM}$; [phosphate buffer] $=0.010 \mathrm{M}$; path length $1.00 \mathrm{~cm} ; V=2.00 \mathrm{~cm}^{3}$; $T=35.0^{\circ} \mathrm{C}$ (pink trace), $25.0^{\circ} \mathrm{C}$ (blue trace), $20.0^{\circ} \mathrm{C}$ (yellow trace), $15.0^{\circ} \mathrm{C}$ (green trace), $10.0^{\circ} \mathrm{C}$ (red trace) and $5.0^{\circ} \mathrm{C}$ (black trace). (For interpretation of the references to colour in this figure legend, the reader is referred to the web version of this article.).

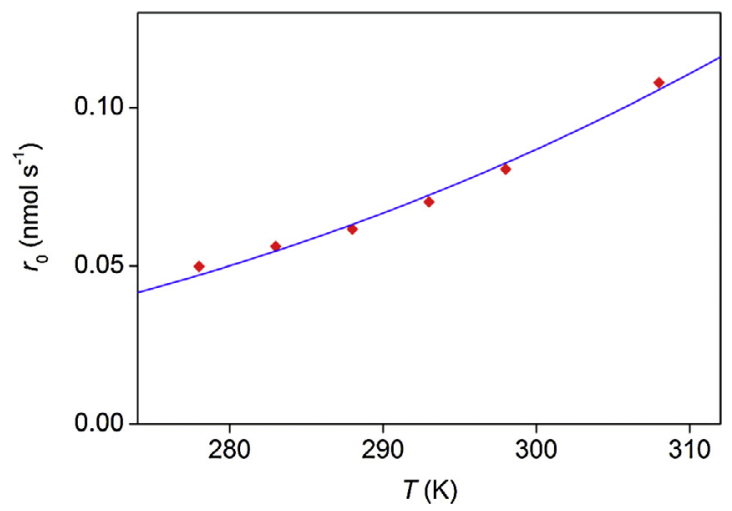

Fig. 10. Dependence of the initial rate on temperature for the photodegradation of TCP. $[\mathrm{TCP}]=0.20 \mathrm{mM}$; [phosphate buffer] $=0.010 \mathrm{M}$; path length $1.000 \mathrm{~cm}$; $V=2.00 \mathrm{~cm}^{3} ; T=5.0-35.0^{\circ} \mathrm{C}$. The solid lines correspond to the fit of data using Eq. (5).

and $A_{2}$ and activation energies of $E_{\mathrm{a} 1}$ and $E_{\mathrm{a} 2}$. The temperature dependence of the quantum yield is simply interpreted in this line of thought as follows:

$\Phi=\frac{k_{2}}{k_{1}+k_{2}}=\frac{A_{2} e^{-E_{a 2} / R T}}{A_{1} e^{-E_{a 1} / R T}+A_{2} e^{-E_{a 2} / R T}}$

The temperature dependence of the initial reaction rate $\left(r_{0}\right.$, now expressed as the time derivative of the amount of substance rather than the $v_{0}$ used earlier, the time derivative of absorbance) is then described as:

$r_{0}=\Phi N=\frac{N}{\left(A_{1} / A_{2}\right) e^{\left(E_{a 2}-E_{a 1}\right) / R T}+1}$

By introducing new parameter combinations, this formula can be written in a much simplified form:

$r_{0}=\frac{r_{\max }}{a e^{b / R T}+1}$

Here, $r_{\max }$ is the maximum rate of the photoreaction that would be detectable in the absence of the radiationless energy loss, $a$ represents the ratio of the two pre-exponential factors, whereas $b$ is the difference of the two activation. The values of $r_{\max }$ could be calculated from the experiments done at $25.0^{\circ} \mathrm{C}$ and was fixed in the evaluation $(1.412 \mathrm{nmol} / \mathrm{s})$. The plot shows that the formula in Eq. (5) gives an excellent interpretation of the observations. Nonlinear least squares fitting was used to obtain the following parameter estimations: $a=0.0046 \pm 0.0021$ and $b=20 \pm 1 \mathrm{~kJ} \mathrm{~mol}^{-1}$. The positive value of $b$ means that the pathway leading to the chemical reaction has a higher activation energy than the radiationless energy loss, whereas $a$ is smaller than 1 , which implies that the pre-exponential factor of the $k_{1}$ pathway is lower than that of $k_{2}$. The excellent fit in Fig. 10 suggests that the simple interpretation proposed in Eq. (2) works well on a quantitative level as well. However, it should also be pointed out that this explanation is quite possible but not inevitable. The observed temperature effect on the photodegradation of TCP degradation might also be due to secondary thermal reactions between the primary active species (i.e. phenoxyl radicals, or phenyl cations) and/or products formed during the overall course of reaction (i.e. $\mathrm{DCQ}+\mathrm{TCP}$ ). These two alternative interpretations could probably be distinguished based on quantitative measurements by determining the quantum yield of the primary photochemical process by a technique with very high time resolution (such as laser flash photolysis or possibly pulse radiolysis).

Based on the findings reported in this paper and previous literature data $[4,33,34,42-44]$, a detailed mechanism with the 


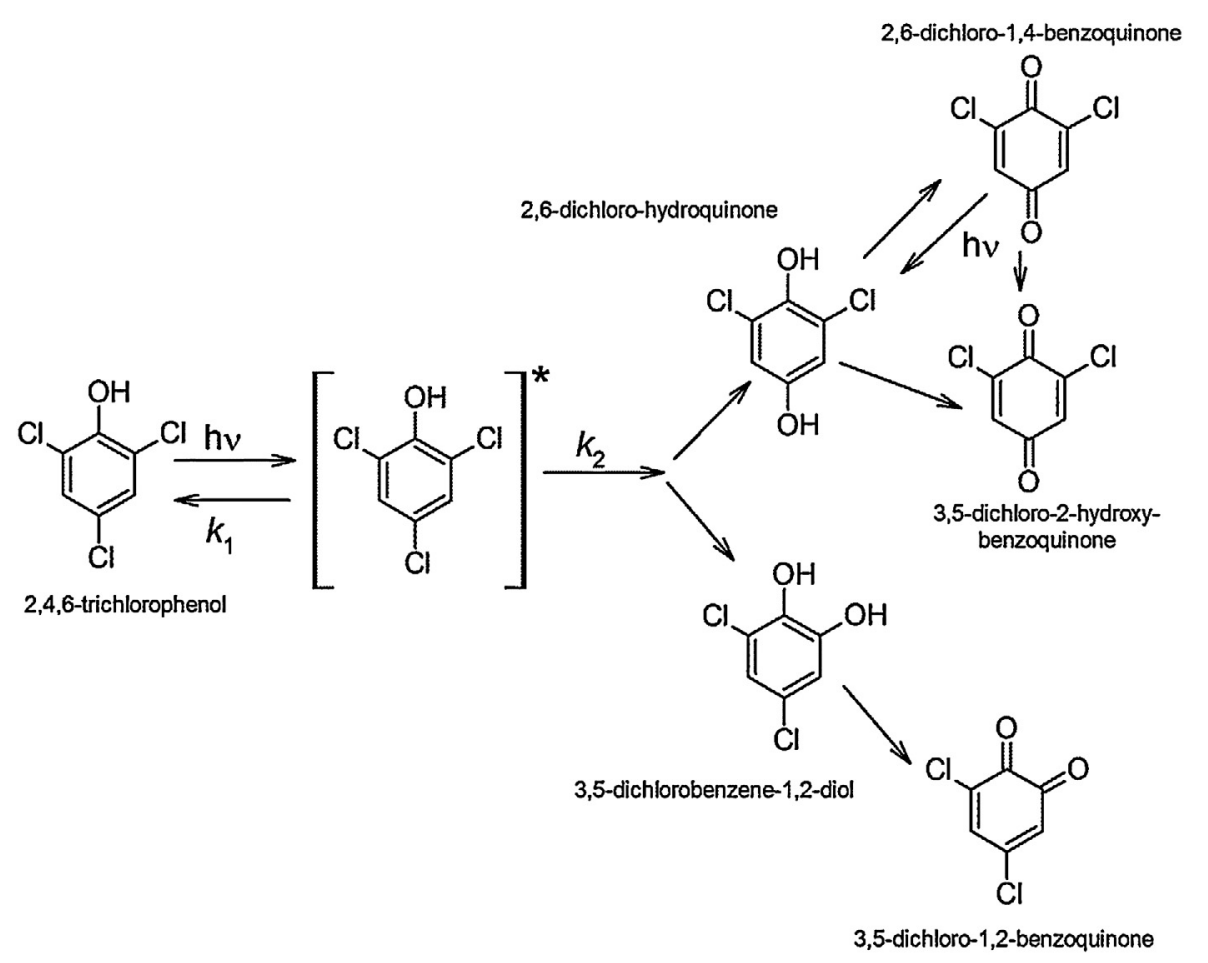

Scheme 1. Proposed reaction scheme for the aqueous photodegradation of 2,4,6-trichlorophenol.

possible chemical structures is shown in Scheme 1. In this mechanism, absorption of light produces the excited form of TCP. As shown in the previous paragraphs, this excited state is involved in two processes: one is the loss of excitation energy without radiation and chemical reactions (its conversion to heat), whereas the other yields new products. Presumably, internal conversion to a triplet state precedes the chemical reactions and the $k_{2}$ may very well represent the unimolecular rate constant of internal conversion. Again, based on the previous knowledge $[4,13,23,33,34,42-44]$, it seems likely that the first direct product of the photoreaction is a hydroquinone, so the process is a substitution of a chlorine atom by a hydroxyl group. This substitution may occur at two positions. Therefore, two products are possible. Each of these hydroquinone products can easily be oxidized to the corresponding quinone. This process is known to be reasonably fast under neutral conditions in the presence of oxygen $[9,24]$. Finally, the 2,6-dichloro-1,4-benzoquinone has a wellknown photoreaction, which produces a hydroxyquinone and the hydroquinone [23].

In addition, Scheme 1 also indicates the possible formation of 3,5-dichlorobenzene-1,2-diol and 3,5-dichloro-1,2-benzoquinone, which could be minor by-products and could explain some of the fine details of experimental observations. For example, 3,5dichloro-1,2-benzoquinone is an isomer of DCQ and might be responsible for the appearance of the peak at retention time $1.62 \mathrm{~min}$ in the chromatogram shown in Fig. 4 and may also cause the initial decrease in absorbance shown in Fig. 2 on the kinetic trace detected at $260 \mathrm{~nm}$.

\section{Conclusion}

2,4,6-Trichlorophenol was almost completely oxidized by a UVvis radiation after $5 \mathrm{~h}$. However, oxidation did not occur when only visible light was used. The products were identified by the UV-vis absorption properties, HPLC and ESI-MS. 2,6-Dichloro-1,4- benzoquinone, 3,5-dichloro-2-hydroxy-1,4-benzoquinone and 2,6-dihclorohydroxyquinone were detected as products of the photodegradation of 2,4,6-trichlorophenol, the second and third probably originating from the independently known aqueous photoreaction of 2,6-dichloro-1,4-benzoquinone. In addition, the formation of 3,5-dichlorobenzene-1,2-diol and 3,5-dichloro-1,2benzoquinone is also possible. The rate of the photochemical process was systematically studied as a function of reactant concentration, reaction volume, illumination ratio and temperature. These observations allowed us to postulate a mechanism, in which the excited form of TCP undergoes radiationless energy loss and a series of chemical reactions in a competitive manner. Because of the competition, the quantum yield of reactant loss shows a modest dependence on temperature, proposing an Arrhenius-type equation interpreted these data reasonably well.

\section{Acknowledgements}

The authors wish to thank the Hungarian Science Foundation (NK 105156) for financial support. The research was supported by the EU and co-financed by the European Social Fund under the project ENVIKUT (TÁMOP-4.2.2.A-11/1/KONV-2012-0043). An anonymous reviewer is also acknowledged for insightful remarks.

\section{Appendix A. Supplementary data}

Supplementary data associated with this article can be found, in the online version, at http://dx.doi.org/10.1016/j. jphotochem.2016.07.025.

\section{References}

[1] J.C. D'Oliveira, C. Minero, E. Pelizzetti, P. Pichat, Photodegradation of dichlorophenols and trichlorophenols in $\mathrm{TiO}_{2}$ aqueous suspensions: kinetic effects of the positions of the $\mathrm{Cl}$ atoms and identification of the intermediates, J. Photochem. Photobiol. A Chem. 72 (1993) 261-267. 
[2] A. Sorokin, B. Meunier, J.L. Séris, Efficient oxidative dechlorination and aromatic ring cleavage of chlorinated phenols catalyzed by iron sulfophthalocyanine, Science 268 (1995) 1163-1166.

[3] Y.S. Shen, Y. Ku, K.C. Lee, The effect of light absorbance on the decomposition of chlorophenols by ultraviolet radiation and u.v./ $\mathrm{H}_{2} \mathrm{O}_{2}$ processes, Water Res. 29 (1995) 907-914.

[4] Y.I. Skurlatov, L.S. Ernestova, E.V. Vichutinskaya, D.P. Samsonov, I.V. Semenova, I.Y. Rod'ko, V.O. Shvidky, R.I. Pervunina, T.J. Kemp, Photochemical transformation of polychlorinated phenols, J. Photochem. Photobiol. A 107 (1997) 207-213.

[5] F.J. Benítez, J. Beltrán-Heredia, J.L. Acero, F.J. Rubio, Chemical decomposition of 2,4,6-trichlorophenol by ozone, fenton's reagent, and UV radiation, Ind. Eng. Chem. Res. 38 (1999) 1341-1349.

[6] F.J. Benitez, J. Beltrán-Heredia, J.L. Acero, F.J. Rubio, Rate constants for the reactions of ozone with chlorophenols in aqueous solutions, J. Hazard. Mater. 79 (2000) 271-285.

[7] S. Sen Gupta, M. Stadler, C.A. Noser, A. Ghosh, B. Steinhoff, D. Lenoir, C. Horwitz, K.W. Schramm, T.J. Collins, Rapid total destruction of chlorophenols by activated hydrogen peroxide, Science 296 (2002) 326-328.

[8] G. Lente, J.H. Espenson, Photoaccelerated oxidation of chlorinated phenols, Chem. Comm. (2003) 1162-1163.

[9] G. Lente, J.H. Espenson, A kinetic study of the early steps in the oxidation of chlorophenols by hydrogen peroxide catalyzed by a water-soluble Iron(III) porphyrin, New J. Chem. 28 (2004) 847-852.

[10] M. Siam, G. Reiter, R. Hunziker, B. Escher, A. Karpfen, A. Simperler, D. Baurecht, U.P. Fringeli, Evidence for heterodimers of 2,4,5-trichlorophenol on planer lipid layers. A FTIR-ATR investigation, Biochim. Biophys. Acta 1664 (2004) 88-99.

[11] G. Lente, J.H. Espenson, Oxidation of 2,4,6-trichlorophenol by hydrogen peroxide. comparison of different iron-based catalysts, Green Chem. 7 (2005) 28-34.

[12] A. Simon, C. Ballai, G. Lente, I. Fábián, Structure-reactivity relationships and substituent effect additivity in the aqueous oxidation of chlorophenols by cerium(IV), New J. Chem. 35 (2011) 235-241.

[13] B.E. Sturgeon, B.J. Battenburg, B.J. Lyon, S. Franzen, Revisiting the peroxidase oxidation of 2,4,6-trihalophenols: ESR detection of radical intermediates, Chem. Res. Toxicol. 24 (2011) 1862-1868.

[14] M. Hu, Y. Wang, Z. Xiong, D. Bi, Y. Zhang, Y. Xu, Iodine-sensitized degradation of 2,4,6-Trichlorophenol under visible light, environ, Sci. Technol. 46 (2012) 9005-9011.

[15] E. Pino, M.V. Encinas, Photocatalytic degradation of chlorophenols on $\mathrm{TiO}_{2}$ 325 mesh and $\mathrm{TiO}_{2}-\mathrm{P} 25$. An extended kinetic study of photodegradation under competitive conditions, J. Photochem. Photobiol. A Chem. 242 (2012) 20-27.

[16] S. Franzen, K. Sasan, B.E. Sturgeon, B.J. Lyon, B.J. Battenburg, H. Gracz, R. Dumariah, R. Ghiladi, Nonphotochemical base-catalyzed hydroxylation of 2,6dichloroquinone by $\mathrm{H}_{2} \mathrm{O}_{2}$ occurs by a radical mechanism, J. Phys. Chem. B 116 (2012) 1666-1676.

[17] H. Ji, F. Chang, X. Hu, Y. Wang, W. Qin, J. Shen, Photocatalytic degradation of 2,4,6-trichlorophenol over g- $\mathrm{C}_{3} \mathrm{~N}_{4}$ under visible light irradiation, Chem. Eng. J. 218 (2013) 183-190.

[18] M. Kurian, D.S. Nair, A.M. Rahnamol, Influence of the synthesis conditions on the catalytic efficiency of $\mathrm{NiFe}_{2} \mathrm{O}_{4}$ and $\mathrm{ZnFe}_{2} \mathrm{O}_{4}$ nanoparticles towards the wet peroxide oxidation of 4-chlorophenol, Reac. Kinet. Mech. Cat. 111 (2014) 591-604.

[19] J.A. Rengifo-Herrera, R.A. Frenzel, M.N. Blanco, L.R. Pizzio, Visible-lightabsorbing mesoporous $\mathrm{TiO}_{2}$ modified with tungstosilicic acid as photocatalyst in the photodegradation of 4-chlorophenol, J. Photochem. Photobiol. A Chem. 289 (2014) 22-30.

[20] J. Rashid, M.A. Barakat, R.M. Mohamed, I.A. Ibrahim, Enhancement of photocatalytic activity of zinc/cobalt spinel oxides by doping with $\mathrm{ZrO}_{2}$ for visible light photocatalytic degradation of 2-chlorophenol in wastewater, J. Photochem. Photobiol. A Chem. 284 (2014) 1-7.

[21] S. Biniak, M. Pakula, A. Swiatkowski, K. Kusmierek, G. Trykowski, Electrooxidation of chlorophenols on powdered carbon electrodes of different porosity, React. Kinet. Catal. Lett. 114 (2015) 369-383.

[22] K. Kusmierek, A. Swiatkowski, The influence of different agitation techniques on the adsorption kinetics of 4-chlorophenol on granular activated carbon, React. Kinet. Mech. Catal. 116 (2015) 261-271.
[23] G. Lente, J.H. Espenson, Photoreduction of 2,6-dichloroquinone in aqueous solution. Use of a diode array spectrophotometer concurrently to drive and detect a photochemical reaction, J. Photochem. Photobiol. A Chem. 163 (2004) 249-258.

[24] É. Józsa, M. Purgel, M. Bihari, P.P. Fehér, G. Sustyák, B. Várnagy, V. Kiss, E. Ladó, K. Ösz, Kinetic studies of hydroxyquinone formation from water soluble benzoquinones, New J. Chem. 38 (2014) 588-597.

[25] I. Kerezsi, G. Lente, I. Fábián, Highly efficient photoinitiation in the cerium(III)catalyzed aqueous autoxidation of sulfur(IV). an example of comprehensive evaluation of photoinduced chain reactions, J. Am. Chem. Soc. 127 (2005) 4785-4793.

[26] I. Fábián, G. Lente, Light induced multistep redox reactions: the diode array spectrophotometer as a photoreactor, Pure Appl. Chem. 82 (2010) 1957-1973.

[27] M. Gombár, É. Józsa, M. Braun, K. Ösz, Construction of a photochemical reacto combining a CCD spectrophotometer and a LED radiation source, Photochem. Photobiol. Sci. 11 (2012) 1592-1595.

[28] T. Lehóczki, É. Józsa, K. Ősz, Ferrioxalate actinometry with online spectrophotometric detection, J. Photochem. Photobiol. A Chem. 251 (2013) 63-68.

[29] J. Kalmár, É. Dóka, G. Lente, I. Fábián, Aqueous photochemical reactions of chloride, bromide, and iodide ions in a diode-array spectrophotometer. Autoinhibition in the photolysis of iodide ion, Dalton Trans. 43 (2014) 4862 4870.

[30] T. Ditrói, J. Kalmár, J.A. Pino-Chamorro, Z. Erdei, G. Lente, I. Fábián, Construction of a multipurpose photochemical reactor with on-line spectrophotometric detection, Photochem. Photobiol. Sci. 15 (2016) 589-594.

[31] SCIENTIST, Version 2.0; Micromath Software: Salt Lake City, UT, USA (1995).

[32] G. Lente, Deterministic Kinetics in Chemistry and Systems Biology, Springer, 2015.

[33] M.P. Yurkova, I.P. Pozdnyakov, V.F. Plyusnin, V.P. Grivin, N.M. Bazhin, A.I. Kruppa, T.A. Maksimova, A mechanistic study of the photodegradation of herbicide 2,4,5-trichlorophenoxyacetic acid in aqueous solution, Photochem. Photobiol. Sci. 12 (2013) 684-689.

[34] I.P. Pozdnyakov, P. Sherin, V. Grivin, V. Plyusnin, Degradation of herbicide 2,4dichlorophenoxybutanoic acid in the photolysis of $[\mathrm{FeOH}] 2+$ and $[\mathrm{Fe}(\mathrm{Ox}) 3] 3-$ complexes: a mechanistic study, Chemosphere 146 (2016) 280-288.

[35] S.E. Braslavsky, Glossary of terms used in photochemistry 3rd edition, Pure Appl. Chem. 79 (2007) 293-465.

[36] C. Gonzalez, J. Pincock, Temperature effects, Arrhenius activation parameters and rate constants for the photochemical reactivity of cyano, boronato, trifluoromethyl and methoxy substituted toluenes in the excited singlet state, Photochem. Photobiol. 82 (2006) 301-309.

[37] N.G.C. Astrath, F.B.G. Astrath, J. Shen, J. Zhou, K.H. Michaelian, C. Fairbridge, L.C. Malacarne, P.R.B. Pedreira, P.A. Santoro, M.L. Baesso, Arrhenius behavior of hydrocarbon fuel photochemical reaction rates by thermal lens spectroscopy, Appl. Phys. Lett. 95 (2009) 191902/1-191902/3.

[38] M. Yamaji, C. Paris, M.T. Miranda, Steady-state and laser flash photolysis studies on photochemical formation of 4-tert-butyl-4'methoxydibenzoylmethane from its derivative via the Norrish Type II reaction in solution, J. Photochem. Photobiol. A: Chem. 209 (2010) 153-157.

[39] T. Asaka, N. Akai, A. Kawai, K. Shibuya, Photochromism of 3-butyl-1-methyl-2phenylazoimidazolium in room temperature ionic liquids, J. Photochem. Photobiol. A: Chem. 209 (2010) 12-18.

[40] S. Rantamäki, E. Tyystjärvi, Analysis of S 2QA - charge recombination with the arrhenius, eyring and marcus theories, J. Photochem. Photobiol. B: Biol. 104 (2011) 292-300.

[41] A. Francois-Heude, E. Richaud, E. Desnoux, X. Colin, A general kinetic model for the photothermal oxidation of polypropylene, J. Photochem. Photobiol. A: Chem. 296 (2015) 48-65.

[42] F. Bonnichon, C. Richard, G. Grabner, Formation of an $\alpha$-ketocarbene by photolysis of aqueous 2-bromophenol, Chem. Commun. 7 (2001) 3-7 (4).

[43] J.P. Da Silva, S. Jockusch, N.J. Turro, Probing the photoreactivity of aryl chlorides with oxygen, Photochem. Photobiol. Sci. 8 (2009) 210-216.

[44] G. Grabner, C. Richard, The Handbook of Environmental Chemistry, in: P. Boule, D.W. Bahnemann, P.K.J. Robertson (Eds.), Environmental Photochemistry, 2, Springer-Verlag Heidelberg, Berlin, 2005, pp. 161-192 (Part M). 